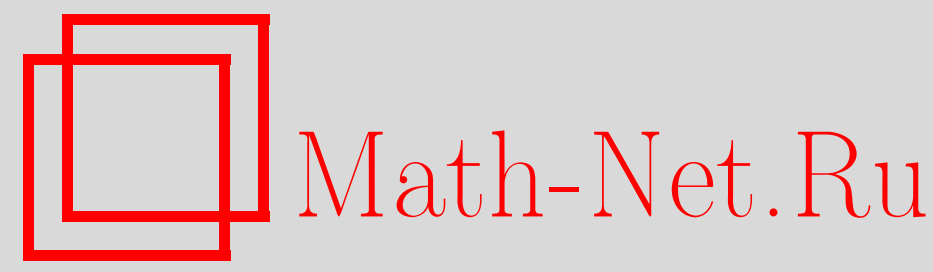

Н. М. Асадуллин, Ф. Х. Мукминов, О классах единственности для нестационарной системы уравнений Стокса в неограниченных областях, Матем. сб., 1996, том 187, номер 3, 3-22

DOI: https://doi.org/10.4213/sm114

Использование Общероссийского математического портала Math-Net.Ru подразумевает, что вы прочитали и согласны с пользовательским соглашением

http://www . mathnet.ru/rus/agreement

Параметры загрузки:

IP : 3.85 .183 .62

26 апреля 2023 г., 05:57:04 
УДК 517.9

Н.М. Асадуллин, Ф.Х. Мукминов

\section{О классах единственности для нестационарной системы уравнений Стокса в неограниченных областях}

Рассматривается задача для нестационарной системы уравнений Стокса в неограниченных областях с условием прилипания на границе. Для областей с некомпактньми границами выделены классы единственности этой задачи, зависящие от геометрии области. Они формулируются в виде ограничений на "рост" скоростей при $|x| \rightarrow \infty$.

Библиография: 9 названий.

Для внешней области и широкого семейства областей с некомпактной границей рассматривается первая смешанная задача для нестационарной системы Стокса. Выделены классы единственности для этой задачи, содержащие, в частности, ограниченные функции.

1. В цилиндре $D^{T}=(0, T) \times \Omega$, где $\Omega \subset \mathbb{R}^{n}, n \geqslant 2,-$ неограниченная область, рассматривается следуюшая задача

$$
\begin{aligned}
& \boldsymbol{u}_{t}=\Delta \boldsymbol{u}-\nabla p+\boldsymbol{f}(t, x), \quad \operatorname{div} \boldsymbol{u}=0, \quad(t, x) \in D^{T} \\
& \left.\boldsymbol{u}\right|_{x \in \partial \Omega}=0, \quad \boldsymbol{u}(0, x)=\boldsymbol{\varphi}(x)
\end{aligned}
$$

Здесь $\boldsymbol{f} \in \boldsymbol{L}_{2, \text { loc }}\left(\bar{D}^{T}\right)$, а начальная функция $\boldsymbol{\varphi}$, принадлежащая пространству $\boldsymbol{L}_{2, \mathrm{loc}}(\bar{\Omega})$, удовлетворяет условию

$$
\int_{\Omega} \varphi \cdot \nabla g d x=0
$$

при любой гладкой функции $g$, финитной в $\bar{\Omega}$. (Как обычно, запись $f \in L_{2, \text { lос }}(Q)$ означает принадлежность $f \in L_{2}(K)$ для любого компакта $K \subset Q ; Q$ - произвольное измеримое множество.) Отметим, что, вообше говоря, постановка задачи (1)-(3) неполная. В работе [3] показано, что если, например, область $\Omega$ - однополостньй гиперболоид, следует также задать поток через его поперечные сечения

$$
\int_{S} \boldsymbol{u}(t, x) \cdot \boldsymbol{n} d S=\widetilde{f}(t), \quad \widetilde{f}(t) \in L_{2}(0, T) .
$$

Для простоты мы ограничиваемся рассмотрением областей, имеющих, как и однополостньй гиперболоид, ровно два выхода на бесконечность.

Второй автор пользовался финансовой поддержкой Российского фонда фундаментальных исследований (грант № 93-011-0273). 
Для некоторых вопросов теории линейных дифференциальных уравнений важную роль играют классы единственности, содержащие, в частности, ограниченные функции. Примером такого класса является класс Тэклинда [8] для уравнения теплопроводности и соответствующее обобщение его на случай второй (первой) смешанной задачи для параболического уравнения (см. [2], [7]). Для системы (1), вообше говоря, класс единственности, содержащий ограниченные функции, должен определяться условиями как на скорости, так и на давление. В самом деле, пара $\boldsymbol{u}(t, x)=-f(t) \boldsymbol{c}, p(t, x)=f^{\prime}(t) \boldsymbol{c} \cdot \boldsymbol{x}, f(0)=0$, является решением задачи Коши для однородной системы уравнений (1). Поэтому интересующий нас класс единственности должен содержать ограничение на рост давления, чтобы исключить приведенное решение. Легко построить аналогичный пример и для внешней задачи, взяв принадлежашее $\boldsymbol{W}_{2}^{0,1}\left(D^{T}\right)$ решение следуюшей задачи

$$
\begin{aligned}
& \boldsymbol{v}_{t}=\Delta \boldsymbol{v}-\nabla q, \quad \operatorname{div} \boldsymbol{v}=0, \quad(t, x) \in D^{T}, \\
& \left.\boldsymbol{v}\right|_{x \in \partial \Omega}=-f(t) \boldsymbol{c}, \quad \boldsymbol{v}(0, x)=0
\end{aligned}
$$

и положив $\overline{\boldsymbol{u}}=\boldsymbol{u}-\boldsymbol{v}$.

Другой вывод из приведенного примера состоит в том, что если функция $f$ не дифференцируема, то градиент давления будет являться обобщенной функцией, что несколько затрудняет формулировку накладьваемых на него требований. В частности, в работе [6] приводится класс единственности для внешней задачи, выделяемый некоторым условием на осредненное давление. Здесь мы приведем этот результат в несколько иной формулировке для области в пространстве произвольной размерности $n \geqslant 2$.

Всюду в работе рассматриваются области с липшицевыми границами. А именно, предполагается, что для каждой точки границы области найдется такая ее окрестность, что пересечение границы с окрестностью в местной системе координат представляется графиком липшицевой функции. При этом соответствуюший кусок границы находится по одну сторону от графика.

Пусть $B_{r}$ - шар радиуса $r$ с центром в начале координат, $\Omega(r)=\Omega \cap B_{r}$, $D^{T}(r)=(0, T) \times \Omega(r)$. Через $\stackrel{\circ}{\boldsymbol{W}}_{2}^{0,1}\left(D^{T}(r)\right)$ будем обозначать пополнение множества $\boldsymbol{C}_{0}^{\infty}\left(D^{T}\right)$ гладких финитных в $D^{T}$ вектор-функций по норме

$$
\|\boldsymbol{u}\|_{0,1}^{2}=\int_{D^{T}(r)}\left(\boldsymbol{u}^{2}+\left|\nabla_{x} \boldsymbol{u}\right|^{2}\right) d x d t .
$$

Будем использовать также следуюшие обозначения.

$$
\nabla \boldsymbol{u}: \nabla \boldsymbol{v}=\sum_{i, j=1}^{n} \frac{\partial u_{i}}{\partial x_{j}} \frac{\partial v_{i}}{\partial x_{j}}, \quad|\nabla \boldsymbol{u}|^{2}=\nabla \boldsymbol{u}: \nabla \boldsymbol{u} .
$$

Пусть $\Omega$ - область, внешняя по отношению к некоторой ограниченной области в пространстве $\mathbb{R}^{n}, n \geqslant 2$. Обобщенным решением внешней задачи (1)-(3) назовем соленоидальную $(\operatorname{div} \boldsymbol{u}=0)$ вектор-функцию $\boldsymbol{u}$, принадлежашую $\stackrel{\circ}{\boldsymbol{W}}_{2}^{0,1}\left(D^{T}(r)\right)$ при всех достаточно больших $r$, и удовлетворяюшую тождеству

$$
\int_{D^{T}}\left(-\boldsymbol{u} \cdot \boldsymbol{v}_{t}+\nabla \boldsymbol{u}: \nabla \boldsymbol{v}-\boldsymbol{f} \cdot \boldsymbol{v}\right) d x d t=\int_{\Omega} \boldsymbol{\varphi} \cdot \boldsymbol{v}(0, x) d x
$$


для всех соленоидальных вектор-функций $\boldsymbol{v} \in \boldsymbol{C}_{0}^{\infty}\left(D_{-1}^{T}\right), D_{a}^{b} \equiv(a, b) \times \Omega$. В этом определении не участвует давление, но оно может быть восстановлено следующим образом. Подставляя в (5) пробные функции вида $\int_{t}^{T} \boldsymbol{v}(\tau, x) d \tau$, для первообразной от скоростей $\boldsymbol{U}(t, x)=\int_{0}^{t} \boldsymbol{u}(\tau, x) d \tau$ легко устанавливаем тождество

$$
\int_{D^{T}} \nabla \boldsymbol{U}: \nabla \boldsymbol{v} d x d t=\int_{D^{T}} \boldsymbol{F} \cdot \boldsymbol{v} d x d t
$$

при тех же пробных функциях $\boldsymbol{v}$, что и выше;

$$
\boldsymbol{F}(t, x) \equiv \int_{0}^{t} \boldsymbol{f}(\tau, x) d \tau+\boldsymbol{\varphi}(x)-\boldsymbol{u}(t, x)
$$

Следовательно, по лемме 2 настоящей работы при почти всех $t \in(0, T)$ вектор $\boldsymbol{U}(t, x)$ вместе с некоторым давлением $P(t, x)$ является решением почти всюду стационарной системы Стокса

$$
-\Delta \boldsymbol{U}=-\nabla P+\boldsymbol{F}(t, x), \quad \operatorname{div} \boldsymbol{U}=0, \quad x \in \Omega
$$

При этом $\nabla P \in \boldsymbol{L}_{2, \text { loc }}(\Omega)$. Очевидно, давление $P$ определяется с точностью до произвольной функции переменной $t$, т.е. фактически имеется целый класс давлений с равньми градиентами. Любую такую функцию $P$ будем называть первообразной от давления, что соответствует действительности, так как если пара $\boldsymbol{u}, p$ является классическим решением задачи (1)-(3), то давления $P(t, x)$ и $\int_{0}^{t} p(\tau, x) d \tau$ отличаются лиш на функцию времени и имеют равные пространственные градиенты. Таким образом, первообразная от давления, в полном соответствии с приведенным выше примером неединственности, имеет регулярный градиент. Этот факт послужил нам мотивировкой для рассмотрения в случае области с некомпактной границей вместо задачи (1)-(4) ее проинтегрированного варианта.

ТЕОРемА 1. Пусть область $\Omega$ в $\mathbb{R}^{n}$ имеет компактную липиичеву гранииу. Пусть и - обобщенное решение однородной задачи (1), (2) с $\boldsymbol{f} \equiv 0, \boldsymbol{\varphi} \equiv 0$, и $P$ - первообразная от давления. Если существует непрерывная монотонно неубьвающая функиия $h$ с расходящимся интегралом $\int^{\infty} d r / h(r)$ такая, что для всех $r \geqslant \operatorname{diam} \partial \Omega$

$$
\int_{0}^{T} \int_{\Omega(r)} \boldsymbol{u}^{2}(t, x) d x \leqslant \exp (r h(r))
$$

u первообразная от давления удовлетворяет при некотором $z \geqslant \operatorname{diam} \partial \Omega$ условию

$$
\lim _{r \rightarrow \infty} r^{-n}\|\nabla P(t)\|_{B_{r} \backslash B_{z}}^{2}=0 \quad \text { npu n.в. } \quad t \in(0, T),
$$

mo $\boldsymbol{u} \equiv 0$. 
Доказательство теоремы 1 проводится так же, как и для соответствующего утверждения в работе [6] $(n=3)$ и поэтому опускается. Надо только "трехмерную" лемму 1 этой работы заменить ее $n$-мерным аналогом - леммой 2 настоящей работы.

Для задачи (1)-(4) в области с некомпактной границей известен класс единственности (см. [5]), формулируемый в терминах только скоростей. С нашей точки зрения этот класс единственности, содержащий, в частности, ограниченные функции, несколько неестественен, так как там накладывается требование $T=\infty$ и формулировка дается в терминах преобразования Фурье-Лапласа от скоростей.

Целью настоящей работы является выделение класса единственности для задачи (1)-(4) в области с некомпактной границей.

Пусть, для простоты изложения, область $\Omega$, как и в работе [5], имеет два выхода на бесконечность, расположенные вдоль оси $O x_{1}$. Предполагается, что множества $\Omega_{a}^{b}=\left\{x \in \Omega: a<x_{1}<b\right\}$ ограничены при всех $a<b$. Далее значения $a=-\infty$ и $b=\infty$ в обозначении $\Omega_{a}^{b}$ будут опускаться. Пусть $l(r), r>0,-$ абсолютно непрерывная монотонно неубывающая функция такая, что $l^{\prime}(r) \leqslant 1-1 / \theta$ при $r>a$, где $a$ и $\theta$ - некоторые числа, большие 1. еПредположим еще, что при $r \geqslant a$ открытые множества $\omega(r)=\Omega_{r-l(r)}^{r}$ связны и удовлетворяют следующему условию. Известно [1], [9], что для каждой ограниченной области $Q$ уравнение

$$
\operatorname{div} \boldsymbol{v}=g, \quad x \in Q, \quad g \in L_{2}(Q), \quad \int_{Q} g d x=0,
$$

имеет решение $\boldsymbol{v} \in \stackrel{\circ}{\boldsymbol{W}}_{2}^{1}(Q)$, удовлетворяюшее оценке

$$
\|\nabla \boldsymbol{v}\|_{Q} \leqslant d(Q)\|g\|_{Q} .
$$

Условие заключается в том, что постоянную $d$ в этом неравенстве можно выбрать единой для каждой области $\omega(r), r \geqslant a$,

$$
\|\nabla \boldsymbol{v}\|_{\omega(r)} \leqslant d\|g\|_{\omega(r)} .
$$

Ради краткости, такое семейство областей $\omega(r)$ будем называть div-ограниченным.

Как следует из результатов работы [1] (см. пункт 6), это условие выполнено, в частности, если области $\omega(r), r \geqslant a$, равномерно звездны относительно некоторых шаров $B(r)$, т.е. отношения $\operatorname{diam} \omega(r) / \operatorname{diam} B(r)$ ограничены постоянной. Если область $\Omega$ такова, что для нее

$$
\Omega_{1}=\left\{x \in \mathbb{R}^{n}:\left|x^{\prime}\right|<x_{1}^{\alpha}, x_{1}>1\right\}
$$

при некотором $\alpha \in(0,1)$, то, очевидно, области $\omega(r), r \geqslant a$, равномерно звездны при достаточно большом $a$, если выбрать $l(r)=r^{\alpha}$. Поэтому такая область удовлетворяет нашему условию.

Нам понадобится еще один пример div-ограниченного семейства областей, вытекающий из результатов той же работы [1]. А именно, для каждой ограниченной области $Q$ с липшицевой границей найдется возрастающая последовательность подобластей с липшицевыми границами $\bar{Q}_{i} \subset Q_{i+1}, i=1,2, \ldots$, такая, что $\bigcup_{i=1}^{\infty} Q^{i}=Q$, и выполнены неравенства

$$
\|\nabla \boldsymbol{v}\|_{Q_{i}} \leqslant d_{1}\|g\|_{Q_{i}}, \quad i=1,2, \ldots
$$


Через $\stackrel{\circ}{V}_{2}^{1}\left(D_{a}^{b}(r)\right), D_{a}^{b}(r)=(a, b) \times \Omega_{-r}^{r}$, обозначим пополнение множества $\boldsymbol{C}_{0}^{\infty}\left(D_{a-1}^{b+1}\right)$ гладких финитных в $D_{a-1}^{b+1}$ векторов по норме

$$
\|\boldsymbol{u}\|_{\boldsymbol{v}, r}^{2}=\int_{D_{a}^{b}(r)} \boldsymbol{u}_{t}^{2} d x d t+\max _{t \in[a, b]} \int_{\Omega_{-r}^{r}}|\nabla \boldsymbol{u}(t, x)|^{2} d x
$$

Через $\boldsymbol{S} \stackrel{\circ}{\boldsymbol{V}}\left(D^{T}\right)$ обозначим пополнение множества соленоидальных векторов из линеала $\boldsymbol{C}_{0}^{\infty}\left(D_{-1}^{T+1}\right)$ по норме $\|\boldsymbol{u}\|_{\boldsymbol{v}} \equiv\|\boldsymbol{u}\|_{\boldsymbol{v}, \infty}$.

Сначала мы определим решение задачи (1)-(4) в предположении $\tilde{f}=0$. Общий случай будет рассмотрен в пункте 4.

Пусть $\boldsymbol{F}(t, x)=\int_{0}^{t} \boldsymbol{f}(\tau, x) d \tau+\varphi(x)$. Обобщенным решением задачи (1)-(4) с $\tilde{f}=0$ назовем вектор $\boldsymbol{u}(t, x)$, если его первообразная $\boldsymbol{U}(t, x)=\int_{0}^{t} \boldsymbol{u}(\tau, x) d \tau$ соленоидальна, при каждом $r>0$ принадлежит пространству $\stackrel{\circ}{V_{2}^{1}}\left(D_{0}^{T}(r)\right)$, имеет нулевой поток через сечения $S_{r}=\left\{x \in \Omega: x_{1}=r\right\}$ и удовлетворяет интегральному тождеству

$$
\int_{D^{T}}\left(\boldsymbol{U}_{t} \cdot \boldsymbol{\eta}+\nabla \boldsymbol{U}: \nabla \boldsymbol{\eta}\right) d x d t=\int_{D^{T}} \boldsymbol{F} \cdot \boldsymbol{\eta} d x d t
$$

при любой соленоидальной вектор-функции $\boldsymbol{\eta} \in \boldsymbol{C}_{0}^{\infty}\left(D^{T}\right)$. Отметим, что классическое решение задачи (1)-(4), принадлежашее соответствующему пространству, будет, очевидно, являться и обобщенным решением этой задачи.

ТЕОРема 2. Пусть область $\Omega$ удовлетворяет описанным выше условиям $u \boldsymbol{u}(t, x)$ - обобщенное решение задачи (1)-(4) с однородными данными $f \equiv 0$, $\varphi \equiv 0, \widetilde{f} \equiv 0$. Положим

$$
h(b, r)=\int_{0}^{T} \int_{\Omega_{b}^{r}} \boldsymbol{u}^{2} d x d t+\sup _{t \in[0, T]} \int_{\Omega_{b}^{r}}|\nabla \boldsymbol{U}|^{2}(t, x) d x .
$$

Существует зависящее только от $d$ и $\theta$ число $\beta>0$ такое, что если

$$
\lim _{k \rightarrow \infty} h\left(0, r_{k}\right) \exp \left(-\int_{a}^{r_{k}} \frac{d \rho}{2 \beta l(\rho)}\right)=0
$$

для некоторой последовательности $r_{k} \rightarrow \infty$, то величина $h(0, \infty)$ конечна. Если жее условие вида (10) выполнено вдоль кажсдого рукава (со своей функиией $l(r))$, то $\boldsymbol{u} \equiv 0$.

Таким образом, теорема 2 выделяет широкий класс единственности, зависящий от геометрии области с некомпактной границей. Отметим, что аналогичный класс единственности в случае первой смешанной задачи для параболического уравнения был выделен в работе [7].

Интересным является вопрос, насколько ограничение "роста скоростей" вида (10) близко к максимально допустимому, при превышении которого появляются примеры неединственности. Нетрудно ответить на него в случае первой смешанной 
задачи для параболического уравнения в областях с некомпактньми границами. А именно, имеется широкое семейство областей, для которых класс единственности теклиндовского типа (задаваемый неравенством из теоремы 1) из работы [2] поглощает тот, которьй выделен в статье [7] условием вида (10). С другой стороны, если область $\Omega$ достаточно быстро сужается на бесконечности, то класс единственности из работы [7] становится шире, чем предложенный в статье [2]. Наша гипотеза состоит в том, что для задачи (1)-(4) ситуация иная: по-видимому, в шшроком классе областей класс единственности, выделяемый условием вида (10), близок к предельному. Однако нам не удалось пока подтвердить этот факт примерами неединственности.

В том случае, когда $\boldsymbol{F} \in \boldsymbol{L}_{2}\left(D^{T}\right)$, методом Галёркина (см., например, [4]) нетрудно доказать существование единственного соленоидального вектора $\boldsymbol{V} \in$ $\boldsymbol{S} \stackrel{\circ}{\boldsymbol{V}}\left(D^{T}\right), \boldsymbol{V}(0, x)=0$, удовлетворяюшего (8). Для него справедливо неравенство

$$
\|\boldsymbol{V}\|_{\boldsymbol{v}} \leqslant 2\|\boldsymbol{F}\|
$$

Здесь и далее через $\|f\|$ будем обозначать норму функции $f$ из пространства $L_{2}(Q)$, где $Q$ - “естественная область определения" этой функции. В пункте 4 приводятся более широкие условия на данные, при которых доказано сушествование решения задачи (1)-(4), принадлежашего выделенному классу единственности.

2. В этом пункте для обобщенного решения задачи (1)-(4) будет выведено неравенство энергетического типа.

Пусть непрерывная функция $\mu(r)$ равна нулю при $r>1$, единице при $r<0$ и линейна в оставшемся интервале. Функция $\xi(x, r)=\mu\left(1+\left(x_{1}-r\right) / l(r)\right)$ непостоянна лишь в слое $\pi(r)=\left\{x \in \mathbb{R}^{n}, r-l(r)<x_{1}<r\right\}$ и

$$
\begin{gathered}
\nabla \xi=\left(\frac{-1}{l(r)}, 0, \ldots, 0\right), \quad x \in \pi(r), \\
\frac{\partial \xi}{\partial r}=\frac{1}{l(r)}+\frac{\left(x_{1}-r\right)}{l^{2}(r)} l^{\prime}(r) \geqslant \frac{1}{\theta l(r)}, \quad x \in \pi(r) .
\end{gathered}
$$

Пусть соленоидальный вектор $\boldsymbol{V}, \boldsymbol{V}(0, x)=0,-$ удовлетворяет тождеству (8), и при всех $t \in(0, T), r>0$ конечна величина

$$
H(t, r)=\int_{0}^{t} \int_{\Omega} \xi \boldsymbol{V}_{t}^{2} d x d t+\frac{1}{2} \int_{\Omega} \xi|\nabla \boldsymbol{V}(t, x)|^{2} d x
$$

Пусть величина $\tilde{h}(R)=\sup _{t \in[0, T]} H(t, R)$ конечна при некотором $R>a$. Предположим также, что поток вектора $\boldsymbol{V}$ через сечения $S_{r}$ равен нулю.

Лемма 1 (основная). Пусть функиия $\boldsymbol{F}(t, x)$, принадлежсащая $\boldsymbol{L}_{2, \operatorname{loc}}\left(\bar{D}^{T}\right)$, равна нулю при $x_{1} \leqslant R$. Найдется число $\beta>1$, зависящее только от $d u$, такое, что при всех $t \in[0, T], r \in[a, R]$ справедливо неравенство

$$
H(t, r) \leqslant C \tilde{h}(R) \exp \left(-\int_{r}^{R} \frac{d \rho}{2 \beta l(\rho)}\right)
$$


Постоянная $C$ зависит только от $T$ и $l(a)$.

Основным этапом доказательства является установление неравенства

$$
H(t, r) \leqslant \beta\left(l(r) H_{r}(t, r)+\frac{1}{l(r)} \int_{0}^{t} H_{r}(\tau, r) d \tau\right)
$$

справедливого для всех $t \in[0, T]$ и $r \in[a, R]$. При этом понадобится следуюшее утверждение.

Лемма 2. Пусть $\boldsymbol{f} \in \boldsymbol{L}_{2, \mathrm{loc}}(\Omega)$, и соленоидальный вектор $\boldsymbol{u}(x) \in \stackrel{\circ}{\boldsymbol{W}}_{2, \text { lос }}^{1}(\Omega)$ удовлетворяет тохдеству

$$
\int_{\Omega}(\nabla \boldsymbol{u}: \nabla \boldsymbol{\eta}-\boldsymbol{f} \cdot \boldsymbol{\eta}) d x=0
$$

при любом соленоидальном векторе $\boldsymbol{\eta} \in \boldsymbol{C}_{0}^{\infty}(\Omega)$. Тогда $\boldsymbol{u} \in \boldsymbol{W}_{2, \mathrm{loc}}^{2}(\Omega)$ u найдется давление $p \in W_{2, \mathrm{loc}}^{1}(\Omega)$ такое, что почти всюду в $\Omega$ справедлива система уравнений

$$
\Delta \boldsymbol{u}=\nabla p+\boldsymbol{f}, \quad \operatorname{div} \boldsymbol{u}=0, \quad x \in \Omega
$$

Доказательство леммы 2 при $n=3$, по-сушеству, имеется в [4]; см. также лемму 1 из [6]. Доказательство, приведенное в последней работе, нетрудно сделать пригодньм при всех $n \geqslant 2$. Для этого вместо симметрического оператора rot следует использовать пару формально сопряженных операторов $A$ и $B$. Оператор $A$ отображает множество $\boldsymbol{M}(\Omega)$ гладких функций со значениями в пространстве кососимметрических матриц порядка $n$ в множество $\boldsymbol{W}(\Omega)$ гладких вектор-функций по формуле

$$
(A \boldsymbol{m})_{j}=\sum_{k=1}^{n} \frac{\partial}{\partial x_{k}} m_{j k}, \quad j=1, \ldots, n, \quad \boldsymbol{m}(x) \in \boldsymbol{M}(\Omega)
$$

Другой оператор $B: \boldsymbol{W}(\Omega) \rightarrow \boldsymbol{M}(\Omega)$ определяется формулами

$$
(B \boldsymbol{w})_{j k}=\frac{\partial}{\partial x_{j}} w_{k}-\frac{\partial}{\partial x_{k}} w_{j}, \quad j, k=1, \ldots, n
$$

В трехмерном случае пространство кососимметрических матриц отождествляется $\mathrm{c} \mathbb{R}^{3}$ по правилу $u_{1}=m_{23}, u_{2}=-m_{13}, u_{3}=m_{12}$, и тогда $A \boldsymbol{m}=\operatorname{rot} \boldsymbol{u}, B \boldsymbol{w}=\operatorname{rot} \boldsymbol{w}$.

Легко видеть, что выполнены обычные свойства “дивергенция ротора равна нулю” и "ротор градиента равен нулю”

$$
\operatorname{div}(A \boldsymbol{m})=0, \quad B(\nabla f)=0, \quad \boldsymbol{m} \in \boldsymbol{M}(\Omega), \quad f \in C_{0}^{\infty}(\Omega)
$$

Более того, выполнено известное равенство

$$
A B \boldsymbol{w}=-\Delta \boldsymbol{w}+\nabla \operatorname{div} \boldsymbol{w}
$$


После названных изменений доказательство “трехмерной” леммы 1 из работы [6] становится пригодным для леммы 2.

Перейдем к установлению неравенства (15).

Зафиксируем произвольное число $t_{0} \in(2 \varepsilon, T-2 \varepsilon), \varepsilon>0$. Пусть $\delta(t)-$ гладкая неотрицательная четная функция с носителем $\operatorname{supp} \delta \subset(-\varepsilon, \varepsilon)$ такая, что $\int_{\text {чим тождество }} \delta(t) d t=1$. Подставив в (8) пробную функцию $\boldsymbol{\eta}(t, x)=\delta\left(t_{0}-t\right) \boldsymbol{v}(x)$, полу-

$$
\int_{\Omega}\left(\boldsymbol{U}_{t}\left(t_{0}\right) \cdot \boldsymbol{v}+\nabla \boldsymbol{U}\left(t_{0}\right): \nabla \boldsymbol{v}\right) d x=\int_{\Omega} \boldsymbol{\Psi}\left(t_{0}\right) \cdot \boldsymbol{v} d x
$$

в котором использованы обозначения $\boldsymbol{U}=\boldsymbol{V} * \delta, \boldsymbol{\Psi}=\boldsymbol{F} * \delta ; \boldsymbol{v} \in \boldsymbol{C}_{0}^{\infty}(\Omega)-$ произвольный соленоидальньй вектор. Следовательно, вектор $\boldsymbol{U}\left(t_{0}\right)$ является обобшенньм решением стационарной системы Стокса с правой частью $\boldsymbol{f}=\boldsymbol{\Psi}\left(t_{0}\right)-$ $\boldsymbol{U}_{t}\left(t_{0}\right)$. По лемме 2 он принадлежит $\boldsymbol{W}_{2, \text { loc }}^{2}(\Omega)$ и вместе с некоторьм давлением $Q \in W_{2, \text { loc }}^{1}(\Omega)$ удовлетворяет почти всюду в $\Omega$ системе уравнений

$$
\boldsymbol{U}_{t}\left(t_{0}\right)-\Delta \boldsymbol{U}\left(t_{0}\right)=\boldsymbol{\Psi}\left(t_{0}\right)-\nabla Q, \quad \operatorname{div} \boldsymbol{U}\left(t_{0}\right)=0 .
$$

Из последней вытекает справедливость тождества

$$
\int_{\Omega}\left(\boldsymbol{U}_{t}\left(t_{0}\right) \cdot \boldsymbol{v}+\nabla \boldsymbol{U}\left(t_{0}\right): \nabla \boldsymbol{v}\right) d x=\int_{\Omega}\left(\mathbf{\Psi}\left(t_{0}\right) \cdot \boldsymbol{v}+Q \operatorname{div} \boldsymbol{v}\right) d x
$$

при любом векторе $\boldsymbol{v} \in \boldsymbol{C}_{0}^{\infty}(\Omega)$. Поскольку давление $Q$ из уравнений Стокса определяется с точностью до произвольной постоянной, мы будем считать, что при некотором фиксированном $r>0$ выполнено равенство $\int_{\omega(r)} Q(x) d x=0$. Более точно, пусть $\omega_{i}$ - такие подобласти области $\omega(r)$, сходящиеся к $\omega(r)$ при $i \rightarrow \infty$, что для них существует единая постоянная $d_{1}$ в неравенствах вида $(7)$, и $c_{i}=\left|\omega_{i}\right|^{-1} \int_{\omega_{i}} Q d x$. Пусть $\boldsymbol{v}^{i} \in \stackrel{\circ}{\boldsymbol{W}_{2}^{1}}\left(\omega_{i+1}\right)$ - решения уравнения

$$
\operatorname{div} \boldsymbol{v}^{i}=\left(Q-c_{i}\right) \chi\left(x ; \omega_{i}\right)
$$

( $\chi$-характеристическая функция), подчиняющиеся оценке

$$
\left\|\nabla \boldsymbol{v}^{i}\right\|_{\omega_{i+1}} \leqslant d_{1}\left\|Q-c_{i}\right\|_{\omega_{i}}
$$

Подставив в (17) $\boldsymbol{v}=\boldsymbol{v}^{i}$ будем иметь оценку

$$
\left\|Q-c_{i}\right\|_{\omega_{i}}^{2} \leqslant\left(\left\|\Psi\left(t_{0}\right)\right\|_{\omega(r)}+\left\|\boldsymbol{U}_{t}\left(t_{0}\right)\right\|_{\omega(r)}\right)\left\|\boldsymbol{v}^{i}\right\|_{\omega_{i+1}}+\left\|\nabla \boldsymbol{U}\left(t_{0}\right)\right\|_{\omega(r)}\left\|\nabla \boldsymbol{v}^{i}\right\|_{\omega_{i+1}} .
$$

Воспользовавшись неравенствами Фридрихса и (18), получим

$$
\left\|Q-c_{i}\right\|_{\omega_{i}}^{2} \leqslant C_{1}(1+l(r)) d_{1}\left(\left\|\Psi\left(t_{0}\right)\right\|_{\omega(r)}+\left\|\boldsymbol{U}_{t}\left(t_{0}\right)\right\|_{\omega(r)}+\left\|\nabla \boldsymbol{U}\left(t_{0}\right)\right\|_{\omega(r)}\right)\left\|Q-c_{i}\right\|_{\omega_{i}} .
$$


Таким образом, можно считать, что ограниченная в $L_{2}(\omega(r))$ последовательность $\left(Q-c_{i}\right) \chi\left(x, \omega_{i}\right)$ имеет слабый предел, который мы и обозначаем через $Q$. Так как $Q \in L_{2}(\omega(r))$, и величина $\tilde{h}(R)$ конечна, то тождество $(17)$ справедливо также и для функции $\boldsymbol{v}=\xi(x) \boldsymbol{U}_{t}\left(t_{0}\right)$. Учитьвая тот факт, что $\boldsymbol{F}(t, x)=0$ при $x_{1}<R$, будем иметь после подстановки этой пробной функции в (17)

$$
\begin{aligned}
\int_{\Omega}\left(\xi\left|\boldsymbol{U}_{t}\left(t_{0}\right)\right|^{2}+\xi \nabla\right. & \left.\boldsymbol{U}\left(t_{0}\right): \nabla \boldsymbol{U}_{t}\left(t_{0}\right)\right) d x \\
& =\int_{\omega(r)}\left(-\boldsymbol{U}_{t}\left(t_{0}\right) \cdot \nabla \boldsymbol{U}\left(t_{0}\right) \cdot \nabla \xi+Q \boldsymbol{U}_{t}\left(t_{0}\right) \cdot \nabla \xi\right) d x
\end{aligned}
$$

Здесь использовано обозначение

$$
\boldsymbol{u} \cdot \nabla \boldsymbol{v} \cdot \nabla \xi=\sum_{i, j=1}^{n} u_{i} \frac{\partial v_{i}}{\partial x_{j}} \frac{\partial \xi}{\partial x_{j}} .
$$

Оценим правую часть в (19), используя соотношение (12):

$$
\left|\int_{\omega(r)} \boldsymbol{U}_{t}\left(t_{0}\right) \cdot \nabla \boldsymbol{U}\left(t_{0}\right) \cdot \nabla \xi d x\right| \leqslant \frac{1}{2} \int_{\omega(r)}\left(\left|\boldsymbol{U}_{t}\left(t_{0}\right)\right|^{2}+\frac{\left|\nabla \boldsymbol{U}\left(t_{0}\right)\right|^{2}}{l^{2}(r)}\right) d x
$$

Для оценки другого интеграла рассмотрим решение уравнения

$$
\operatorname{div} \boldsymbol{w}=\frac{\partial U_{1}\left(t_{0}, x\right)}{\partial t}, \quad x \in \omega(r)
$$

принадлежашее $\stackrel{\circ}{\boldsymbol{W}} \underset{2}{1}(\omega(r))$ и удовлетворяющее неравенству

$$
\|\nabla \boldsymbol{w}\| \leqslant d\left\|U_{1, t}\left(t_{0}\right)\right\|_{\omega(r)} .
$$

Условие разрешимости уравнения (20) выполнено, так как вектор $\boldsymbol{V}$ имеет нулевой поток через сечения $S_{r}$, что влечет за собой равенство нулю и потока вектора $\boldsymbol{U}_{t}\left(t_{0}\right)$ :

$$
\int_{S_{r}} U_{1, t}\left(t_{0}, x\right) d S=\int_{S_{r}} \boldsymbol{U}_{t}\left(t_{0}\right) \cdot \boldsymbol{n} d S=0 \quad \text { для п.в. } r .
$$

По неравенству Фридрихса

$$
\|\boldsymbol{w}\|_{\omega(r)} \leqslant b l(r)\|\nabla \boldsymbol{w}\|_{\omega(r)} \leqslant b d l(r)\left\|\boldsymbol{U}_{t}\left(t_{0}\right)\right\|_{\omega(r)},
$$

$b$ - абсолютная постоянная. Пользуясь тождеством (17), можем записать

$$
\begin{aligned}
\left|\int_{\omega(r)} Q \boldsymbol{U}_{t}\left(t_{0}\right) \cdot \nabla \xi d x\right| & =\frac{1}{l(r)}\left|\int_{\omega(r)} Q \operatorname{div} \boldsymbol{w} d x\right| \\
& =\left|\frac{1}{l(r)} \int_{\omega(r)}\left(\boldsymbol{U}_{t}\left(t_{0}\right) \cdot \boldsymbol{w}+\nabla \boldsymbol{U}\left(t_{0}\right): \nabla \boldsymbol{w}\right) d x\right| \\
& \leqslant b d\left\|\boldsymbol{U}_{t}\left(t_{0}\right)\right\|_{\omega(r)}^{2}+\frac{d\left\|\nabla \boldsymbol{U}\left(t_{0}\right)\right\|_{\omega(r)}\left\|\boldsymbol{U}_{t}\left(t_{0}\right)\right\|_{\omega(r)}}{l(r)} \\
& \leqslant\left(b d+\frac{d}{2}\right)\left\|\boldsymbol{U}_{t}\left(t_{0}\right)\right\|_{\omega(r)}^{2}+\frac{d\left\|\nabla \boldsymbol{U}\left(t_{0}\right)\right\|_{\omega(r)}^{2}}{2 l^{2}(r)} .
\end{aligned}
$$


Итак,

$$
\begin{aligned}
\int_{\Omega} \xi\left(\left|\boldsymbol{U}_{t}\left(t_{0}\right)\right|^{2}+\nabla \boldsymbol{U}\left(t_{0}\right):\right. & \left.\nabla \boldsymbol{U}_{t}\left(t_{0}\right)\right) d x \\
& \leqslant(1+d+b d)\left(\left\|\boldsymbol{U}_{t}\left(t_{0}\right)\right\|_{\omega(r)}^{2}+\frac{\left\|\nabla \boldsymbol{U}\left(t_{0}\right)\right\|_{\omega(r)}^{2}}{2 l^{2}(r)}\right) .
\end{aligned}
$$

Выберем числа $t, s$ из интервала $(2 \varepsilon, T-2 \varepsilon)$ и проинтегрируем последнее по $t_{0} \in(s, t)$. Получим

$$
\begin{aligned}
& \int_{s}^{t} \int_{\Omega} \xi\left|\boldsymbol{U}_{t}\right|^{2} d x d \tau+\frac{1}{2} \int_{\Omega} \xi|\nabla \boldsymbol{U}(t)|^{2} d x \\
& \quad \leqslant \frac{1}{2} \int_{\Omega} \xi|\nabla \boldsymbol{U}(s)|^{2} d x+(1+d+b d)\left(\int_{s}^{t} \int_{\omega(r)}\left(\boldsymbol{U}_{t}^{2}+\frac{|\nabla \boldsymbol{U}|^{2}}{2 l^{2}(r)}\right) d x d \tau\right) .
\end{aligned}
$$

Переходя в последнем к пределу по $\delta$-образной последовательности $\delta_{k}(t), k \rightarrow \infty$, будем иметь

$$
\begin{aligned}
& \int_{s}^{t} \int_{\Omega} \xi\left|\boldsymbol{V}_{t}\right|^{2} d x d \tau+\frac{1}{2} \int_{\Omega} \xi|\nabla \boldsymbol{V}(t)|^{2} d x \\
& \quad \leqslant \frac{1}{2} \int_{\Omega} \xi|\nabla \boldsymbol{V}(s)|^{2} d x+(1+d+b d)\left(\int_{s}^{t} \int_{\omega(r)}\left(\boldsymbol{V}_{t}^{2}+\frac{|\nabla \boldsymbol{V}|^{2}}{2 l^{2}(r)}\right) d x d \tau\right) .
\end{aligned}
$$

Наконец, предельньй переход $s \rightarrow 0$ дает неравенство

$$
H(t, r) \leqslant \widetilde{\beta} \int_{0}^{t} \int_{\omega(r)}\left(\boldsymbol{V}_{t}^{2}+\frac{|\nabla \boldsymbol{V}|^{2}}{2 l^{2}(r)}\right) d x d \tau,
$$

где $\widetilde{\beta}$ зависит только от $d$. Заметим, что ввиду (13)

$$
\begin{gathered}
\int_{0}^{t} \int_{\omega(r)} \boldsymbol{V}_{t}^{2} d x d \tau \leqslant \theta \frac{\partial H(t, r)}{\partial r} \\
\int_{0}^{t} \int_{\omega(r)}|\nabla \boldsymbol{V}|^{2} d x d \tau \leqslant 2 \theta l(r) \int_{0}^{t} \frac{\partial H(\tau, r)}{\partial r} d \tau .
\end{gathered}
$$

Поэтому из (21) следует (15).

Сделаем в (15) замену переменной $r \rightarrow s$, где функция $s(r)$ является решением задачи Коши: $s^{\prime}=1 / \beta l(r), s(a)=0$. Для функции $G(t, s)=H(t, r(s))$, с учетом монотонного роста функции $l(r)$, будем иметь неравенство

$$
G(t, s) \leqslant G_{s}(t, s)+\int_{0}^{t} G_{s}(\tau, s) d \tau / l^{2}(a) .
$$

Интегрирование по $s$ и отбрасывание отрицательных слагаемых в правой части дает соотношение

$$
\int_{0}^{s} G(t, \rho) d \rho \leqslant G(t, s)+\int_{0}^{t} G(\tau, s) d \tau / l^{2}(a) .
$$


После $2 n$-кратного интегрирования приходим к неравенству

$$
\int_{0}^{s} \frac{(s-\rho)^{2 n-1}}{(2 n-1) !} G(t, \rho) d \rho \leqslant(1+\boldsymbol{I})^{2 n} G,
$$

где через $\boldsymbol{I}$ обозначен оператор

$$
\boldsymbol{I} G=\int_{0}^{t} G(\tau, s) d s / l^{2}(a)
$$

Поскольку $G(t, s)=H(t, r) \leqslant \tilde{h}(R)$ при $t \in[0, T], r \in[a, R]$, то

$$
(1+\boldsymbol{I})^{2 n} G \leqslant \tilde{h}(R) C_{2 n}^{n}\left(1+\boldsymbol{I}(1)+\boldsymbol{I}^{2}(1)+\cdots\right)=\tilde{h}(R) C_{2 n}^{n} \exp \left(\frac{T}{l^{2}(a)}\right) .
$$

С другой стороны, ввиду неубывания функции $G(t, s)$ по переменной $s$

$$
\int_{0}^{s_{1}} \frac{\left(s_{1}-\rho\right)^{2 n-1}}{(2 n-1) !} G(t, \rho) d \rho \geqslant G(t, s) \int_{s}^{s_{1}} \frac{\left(s_{1}-\rho\right)^{2 n-1}}{(2 n-1) !} d \rho=\frac{\left(s_{1}-s\right)^{2 n}}{2 n !} G(t, s) .
$$

Следовательно,

$$
G(t, s) \leqslant c_{2}(l(a), T) \tilde{h}(r) \frac{2 n !}{\left(s_{1}-s\right)^{2 n}} C_{2 n}^{n} .
$$

Применив формулу Стирлинга, получим

$$
G(t, s) \leqslant c_{3} \tilde{h}(R)\left(\frac{4 n}{e\left(s_{1}-s\right)}\right)^{2 n} .
$$

Положим $n=\left[\frac{s_{1}-s}{4}\right] \geqslant \frac{s_{1}-s}{4}-1$. Будем иметь

$$
G(t, s) \leqslant c_{4} \tilde{h}(R) \exp \left(-\frac{s_{1}-s}{2}\right) .
$$

При $s_{1}=s(R)$, перейдя к переменной $r$, получим неравенство (14). Лемма 1 доказана.

3. Доказательство теоремы 2. Применим лемму 1 к вектору $\boldsymbol{V}=\boldsymbol{U}$, предполагая сначала, что $\tilde{h}(r)<\infty$ при некотором $r>0$. Пользуясь (10), перейдем в (14) к пределу по последовательности $R=r_{k}, k \rightarrow \infty$. Получим $H(t, r)=0$. Ввиду произвольности $r>a$, отсюда следует, что $\boldsymbol{U} \equiv \boldsymbol{V}=0$, а также $\boldsymbol{u}=0$. Отметим, что в приведенных рассуждениях вместо (10) было использовано, вообше говоря, более слабое условие

$$
\lim _{r_{k} \rightarrow \infty} \tilde{h}\left(r_{k}\right) \exp \left(-\int_{a}^{r_{k}} \frac{d \rho}{2 \beta l(\rho)}\right)=0 .
$$

Сведем общий случай к рассмотренному, взяв срезку $\zeta \boldsymbol{U}$ с некоторой функцией $\zeta\left(x_{1}\right)$ и ликвидировав возникшую в уравнениях неоднородность вычитанием подходящей функции. 
Вектор $\boldsymbol{U}$, по определению обобшенного решения задачи (1)-(4), удовлетворяет однородному тождеству (8), $\boldsymbol{F}=0$. Переходя, при необходимости, к первообразной, можно считать, что функции $\boldsymbol{U}_{t}(t), \nabla \boldsymbol{U}(t)$ непрерывны по $t$ в норме $\boldsymbol{L}_{2}(K)$ для любого компакта $K \subset \bar{\Omega}$. Тогда, аналогично тому как это сделано в пункте 2, устанавливаем, что вектор $\boldsymbol{U}$ вместе с некоторым давлением $Q$ является решением почти всюду системы уравнений $\boldsymbol{U}_{t}=\Delta \boldsymbol{U}-\nabla Q$. При этом $\Delta \boldsymbol{U}(t), \nabla Q(t) \in \boldsymbol{L}_{2, \operatorname{loc}}(\Omega)$, и $Q(t) \in \boldsymbol{L}_{2, \operatorname{loc}}(\bar{\Omega})$.

Пусть $\zeta\left(x_{1}\right)$ - гладкая срезаюшая функция, равная нулю при $x_{1}<-b$, единище при $x_{1}>1-b ; b$ - достаточно большое положительное число. Положим $\widetilde{\boldsymbol{V}}=\zeta \boldsymbol{U}-\boldsymbol{w}, \widetilde{Q}=\zeta Q$. Вектор $\boldsymbol{w}(t, x)$ подбирается так, чтобы вектор $\widetilde{\boldsymbol{V}}$ был соленоидальньм:

$$
\operatorname{div} \boldsymbol{w}=\boldsymbol{U} \cdot \nabla \zeta, \quad x \in \omega=\left\{x \in \Omega:-b<x_{1}<1-b\right\}
$$

Условие разрешимости $\int_{\omega} \boldsymbol{U} \cdot \nabla \zeta d x=0$ выполнено, так как вектор $\boldsymbol{U}$ имеет нулевой поток через сечения $S_{r}$. Поскольку $\omega$ - область с липшицевой границей и $\boldsymbol{U} \cdot \nabla \zeta \in \stackrel{\circ}{\boldsymbol{W}}_{2}^{1}(\omega)$, то (см. [1]) существует решение уравнения (22), принадлежащее $\stackrel{\circ}{\boldsymbol{W}}_{2}^{2}(\Omega)$ при каждом $t \in[0, T]$. Так как оно определяется линейным оператором $\boldsymbol{w}=\boldsymbol{R}(\boldsymbol{U} \cdot \nabla \zeta)$ (см. [1]) и функция $\boldsymbol{U}_{t}(t)$ непрерывна по $t$ в $\boldsymbol{L}_{2}(\omega)$, то $\boldsymbol{w}_{t} \in \boldsymbol{L}_{2}(\omega)$. Далее считаем вектор $\boldsymbol{w}$ продолженным нулем вне $\omega$.

Установим уравнения, которьм удовлетворяют новые скорости и давление

$$
\widetilde{\boldsymbol{V}}_{t}-\Delta \widetilde{\boldsymbol{V}}+\nabla \widetilde{Q}=-2 \nabla \zeta \cdot \nabla \boldsymbol{U}-\boldsymbol{U} \Delta \zeta+Q \nabla \zeta+\Delta \boldsymbol{w}-\boldsymbol{w}_{t}
$$

Правую часть в $(23)$ обозначим через $\boldsymbol{F}$. Она принадлежит $\boldsymbol{L}_{2}\left(D^{T}\right)$. Поэтому, согласно пункту 2 , найдется соленоидальный вектор $\boldsymbol{W} \in \underset{\boldsymbol{S}}{\boldsymbol{V}}\left(D^{T}\right), \boldsymbol{W}(0, x)=0$, удовлетворяющий тождеству (8). Легко проследить, что вектор $\boldsymbol{Z}=\widetilde{\boldsymbol{V}}-\boldsymbol{W}$ соленоидален, удовлетворяет тождеству (8) с $\boldsymbol{F}=0$ и для него конечны величины $\tilde{h}(r)$ при каждом $r>0$. Более того, останется выполненным условие $\left(9^{\prime}\right)$. По предыдущему устанавливаем, что $\boldsymbol{Z}=0$, т.е. $\widetilde{\boldsymbol{V}}=\boldsymbol{W}$. Таким образом, для вектора $\boldsymbol{U}$ конечна величина $h(0, \infty)$, что доказывает первое утверждение теоремы.

Аналогично, из условия вида (10) вдоль другого рукава, получаем конечность величины $h(-\infty, 0)$. Тогда для вектора $\boldsymbol{U}$ конечны величины $\tilde{h}(r)$ при каждом $r>0$, что, как было установлено выше, приводит к равенству $\boldsymbol{u}=0$. Теорема 2 доказана.

4. В этом пункте приводятся условия сушествования решения задачи (1)-(4), принадлежашего выделяемому теоремой 2 классу единственности.

Рассмотрим функцию $\boldsymbol{w} \in \stackrel{\circ}{\boldsymbol{W}}_{2,1 \text {, }}^{1}(\bar{\Omega})$, удовлетворяющую условиям

$$
\int_{S_{r}} \boldsymbol{w} \cdot \boldsymbol{n} d S=1, \quad \operatorname{div} \boldsymbol{w}=0
$$

и тождеству

$$
\int_{\Omega} \nabla \boldsymbol{w} \cdot \nabla \boldsymbol{\eta} d x=0
$$


для любой соленоидальной вектор-функции $\boldsymbol{\eta} \in \boldsymbol{C}_{0}^{\infty}(\Omega)$. Об условиях существования такой функции, удовлетворяющей дополнительно некоторым интегральньм оценкам, будет сказано ниже, в пункте 5 .

Очевидно, что при условиях, наложенных на данные задачи (1)-(4) и вектор $\boldsymbol{w}$, следующий вектор

$$
\boldsymbol{F}(t, x)=\int_{0}^{t} \boldsymbol{f}(\tau, x) d \tau+\varphi(x)-\tilde{f}(t) \boldsymbol{w}(x)
$$

принадлежит $\boldsymbol{L}_{2, \mathrm{loc}}\left(\bar{D}^{T}\right)$. Обобщенным решением задачи (1)-(4) назовем такой вектор $\boldsymbol{u}(t, x)$, что его первообразная $\boldsymbol{U}(t, x)=\int_{0}^{t} \boldsymbol{u}(\tau, x) d \tau$ имеет вид $\boldsymbol{U}(t, x)=$ $\boldsymbol{V}(t, x)+\left(\int_{0}^{t} \tilde{f}(\tau) d \tau\right) \boldsymbol{w}(x)$, где соленоидальный вектор $\boldsymbol{V}, \boldsymbol{V}(0, x)=0$, при каждом $r>0$ принадлежит пространству $\stackrel{\circ}{{ }^{1}} \underset{2}{1}\left(D_{0}^{T}(r)\right)$, имеет нулевой поток через сечения $S_{r}$ и удовлетворяет интегральному тождеству (8). Такое определение корректно, так как не зависит от выбора функции $\boldsymbol{w}$.

В следуюшем утверждении $\beta=(1+d+b d) \theta$ - постоянная из леммы 1 .

ТЕОРемА 3. Пусть область $\Omega$ удовлетворяет тем жее условиям, что и в теореме 2. Пусть для функиии $\boldsymbol{F}(t, x)$ конечны интеграль

$$
\int_{0}^{T} \int_{\Omega^{a}} \boldsymbol{F}^{2} d x d t
$$

$u$

$$
\int_{0}^{T} \int_{\Omega_{a}} \exp \left(-\int_{a}^{x_{1}} \frac{d \rho}{2 \beta l(\rho)}\right) \boldsymbol{F}^{2} d x d t
$$

Тогда существует единственный соленоидальный вектор $\boldsymbol{V}, \boldsymbol{V}(0, x)=0$, принадлежсащий пространствам $\stackrel{\circ}{V}_{2}^{1}\left(D^{T}(r)\right), r>0$, для которого конечньи интеграль $\tilde{h}(r), r>0$, справедливо тожсдество (8) и выполнено условие $\left(10^{\prime}\right)$ с некоторой возрастающей последовательностью $r_{k} \rightarrow \infty$.

ДоКАЗАТЕЛЬСТво. Положим $r_{1}=a$, а остальные члены последовательности выберем так, чтобы

$$
r_{k+1}=l\left(r_{k}\right)+r_{k}
$$

Пусть $\boldsymbol{F}^{1}(t, x)=\chi\left(x_{1} ;-\infty, a\right) \boldsymbol{F}, \chi-$ характеристическая функция интервала $(-\infty, a)$, и $\boldsymbol{F}^{k}=\chi\left(x_{1} ; r_{k-1}, r_{k}\right) \boldsymbol{F}, k=2,3, \ldots$ Так как $\boldsymbol{F}^{k} \in \boldsymbol{L}_{2}\left(D^{T}\right)$, то как отмечалось во введении, сушествуют соленоидальные векторы $\boldsymbol{V}^{k}, \boldsymbol{V}^{k}(0, x)=0$, удовлетворяющие тождеству (8) с $\boldsymbol{F}=\boldsymbol{F}^{k}$, принадлежащие пространству $\boldsymbol{S} \stackrel{\circ}{\boldsymbol{V}}\left(D^{T}\right)$ и подчиняющиеся оценкам

$$
\left\|\boldsymbol{V}^{k}\right\|_{\boldsymbol{v}} \leqslant 2\left\|\boldsymbol{F}^{k}\right\|
$$

Докажем, что сумма $\sum_{k=1}^{\infty} \boldsymbol{V}^{k}$ сходится в каждом из пространств $\stackrel{\circ}{\boldsymbol{V}_{2}^{1}}\left(D^{T}(r)\right)$, $r>0$, и определяет вектор, удовлетворяющий описанным в теореме 3 требованиศM. 
По функциям $\boldsymbol{V}^{k}$ определим, как в пункте 2 , функции $H_{k}(t, r)$ и $\tilde{h}_{k}(r)$. Для каждого $k=3,4, \ldots$ функции $\boldsymbol{F}^{k}$ равны нулю при $x \leqslant r_{k-1}$, следовательно, векторы $\boldsymbol{V}^{k}$ удовлетворяют условиям основной леммы. Справедливы неравенства вида (14)

$$
H_{k}(t, r) \leqslant c \tilde{h}_{k}(R) \exp \left(-\int_{r}^{R} \frac{d \rho}{2 \beta l(\rho)}\right), \quad a<r<R<r_{k-1} .
$$

Очевидно, $\tilde{h}_{k}(R) \leqslant 4\left\|\boldsymbol{F}^{k}\right\|^{2}, H_{k}(t, r) \leqslant 4\left\|\boldsymbol{F}^{k}\right\|^{2}, k=1,2, \ldots$ Поэтому

$$
\begin{aligned}
\sum_{k=i}^{\infty} H_{k+1}\left(t, r_{i}\right) & \leqslant \sum_{k=i}^{\infty} 4 c\left\|\boldsymbol{F}^{k+1}\right\|^{2} \exp \left(-\int_{r_{i}}^{r_{k}} \frac{d \rho}{2 \beta l(\rho)}\right) \\
& =4 c \exp \left(\int_{a}^{r_{i}} \frac{d \rho}{2 \beta l(\rho)}\right) \sum_{k=i}\left\|\boldsymbol{F}^{k+1}\right\|^{2} \exp \left(-\int_{a}^{r_{k}} \frac{d \rho}{2 \beta l(\rho)}\right) .
\end{aligned}
$$

По построению последовательности $r_{k}$ имеем

$$
\int_{r_{k}}^{r_{k+1}} \frac{d \rho}{l(\rho)} \leqslant \frac{r_{k+1}-r_{k}}{l\left(r_{k}\right)}=1
$$

Следовательно,

$$
\begin{aligned}
& \sum_{k=i}^{\infty} H_{k+1}\left(t, r_{i}\right) \\
& \quad \leqslant c_{1}(l(a), T, \beta) \exp \left(\int_{a}^{r_{i}} \frac{d \rho}{2 \beta l(\rho)}\right) \sum_{k=i}^{\infty}\left\|\boldsymbol{F}^{k+1}\right\|^{2} \exp \left(-\int_{a}^{r_{k+1}} \frac{d \rho}{2 \beta l(\rho)}\right) \\
& \quad \leqslant c_{1} \exp \left(\int_{a}^{r_{i}} \frac{d \rho}{2 \beta l(\rho)}\right) \int_{0}^{T} \int_{\Omega_{r_{i}}} \boldsymbol{F}^{2} \exp \left(-\int_{a}^{x_{1}} \frac{d \rho}{2 \beta l(\rho)}\right) d x d t
\end{aligned}
$$

Это вместе с условием конечности интеграла (26) влечет соотношение

$$
\sum_{k=i}^{\infty} H_{k+1}\left(t, r_{i}\right)=\varepsilon_{i} \exp \left(\int_{a}^{r_{i}} \frac{d \rho}{2 \beta l(\rho)}\right)
$$

где $\varepsilon_{i}$ - стремящаяся к нулю числовая последовательность.

Далее,

$$
\sum_{k=1}^{i} H_{k}\left(t, r_{i}\right) \leqslant \sum_{k=1}^{i} 4\left\|\boldsymbol{F}^{k}\right\|^{2}=4 \int_{0}^{T} \int_{\Omega^{r_{i}}} \boldsymbol{F}^{2} d x d t .
$$

Отсюда, как показано ниже, вытекает соотношение

$$
\sum_{k=1}^{i} H_{k}\left(t, r_{i}\right)=\delta_{i} \exp \left(\int_{a}^{r_{i}} \frac{d \rho}{2 \beta l(\rho)}\right), \quad \delta_{i} \rightarrow 0 .
$$


ЛЕмма 3. Пусть $\lambda(r)$ - монотонно стремящаяся $\kappa$ бесконечности при $r \rightarrow \infty$ функиия, равная 1 при $r \in(-\infty, a]$, и $p(r) \in L_{1}(-\infty, \infty)$ - неотрииательная функиия.

Тогда

$$
\lim _{r \rightarrow \infty} \lambda^{-1}(r) \int_{-\infty}^{r} \lambda(\rho) p(\rho) d \rho=0
$$

ДокАЗАТЕЛЬСТво.

$$
\lambda^{-1}(r) \int_{-\infty}^{r} \lambda(\rho) p(\rho) d \rho \leqslant \frac{\lambda(\tau)}{\lambda(r)} \int_{-\infty}^{\tau} p(\rho) d \rho+\int_{\tau}^{r} p(\rho) d \rho
$$

Для завершения доказательства леммы 3 достаточно выбрать стремящуюся к бесконечности функцию $\tau(r)$ такую, что

$$
\lim _{r \rightarrow \infty} \frac{\lambda(\tau(r))}{\lambda(r)}=0
$$

Чтобы получить (29), мы полагаем

$$
\lambda(r)=\exp \left(\int_{a}^{r} \frac{d \rho}{2 \beta l(\rho)}\right), \quad r>a, \quad p(r)=\int_{0}^{T} \int_{S_{r}} \boldsymbol{F}^{2}\left(t,\left(r, x^{\prime}\right)\right) d x^{\prime} d t
$$

и применяем лемму 3. Из соотношений $(28),(29)$ следует, что функция $\boldsymbol{V}=\sum_{k=1}^{\infty} \boldsymbol{V}^{k}$ принадлежит пространствам $\stackrel{\circ}{\boldsymbol{V}} \underset{2}{1}\left(\boldsymbol{D}^{T}(r)\right), r>0$, удовлетворяет тождеству (8) и для нее справедливы неравенства

$$
H\left(t, r_{i}\right) \leqslant\left(\varepsilon_{i}+\delta_{i}\right) \exp \left(\int_{a}^{r_{i}} \frac{d \rho}{2 \beta l(\rho)}\right), \quad i=1,2, \ldots
$$

обеспечивающие принадлежность функции $\boldsymbol{V}$ классу единственности $\left(10^{\prime}\right)$. Теорема 3 доказана.

СЛЕДСТВИЕ. Пусть область $\Omega$ удовлетворяет тем же условиям, что и в теореме 2 по правому рукаву, и аналогичным условиям с функиией $l_{-}$по левому рукаву. Пусть функиии $\boldsymbol{f}, \boldsymbol{\varphi}, \boldsymbol{w}$ удовлетворяют условию конечности интегралов вида (26) вдоль каждого рукава области $\Omega$, , кроме того, $\tilde{f}(t) \in L_{2}(0, T)$. Тогда существует единственное обобщенное решение задачи (1)-(4), принадлежащее классу единственности, въделяемому условием вида $\left(10^{\prime}\right)$ вдоль кажсдого рукава; в последнем $\tilde{h}(r)$ вычисляется по функчии $\boldsymbol{U}$.

5. В этом пункте решается вопрос о сушествовании функции $\boldsymbol{w}$, удовлетворяющей условиям (24), тождеству (25), такой, что для нее конечны интегралы вида (26). Ради краткости будем использовать термин “задача $(24),(25)$ ”, хотя, вообще говоря, существует много функций, удовлетворяющих условиям $(24),(25)$. 
ТЕОРемА 4. Пусть область $\Omega$ удовлетворяет тем жсе условиям, что и в теореме 2 по правому рукаву, и аналогичным условиям с функиией $l_{-}$по левому рукаву. Пусть существует вектор-функиия $\boldsymbol{f} \in \stackrel{\circ}{\boldsymbol{W}}_{2, \text { lос }}^{1}(\bar{\Omega})$ с единичны.м потоком через сечения $S_{r}$, удовлетворяющая условию конечности интегра-

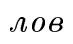

$$
\int_{\Omega_{a}}|\nabla \boldsymbol{f}|^{2} \exp \left(-\int_{a}^{x_{1}} \frac{d \rho}{b l(\rho)}\right) d x+\int_{\Omega^{-a}}|\nabla \boldsymbol{f}|^{2} \exp \left(-\int_{x_{1}}^{-a} \frac{d \rho}{b l_{-}(\rho)}\right) d x<\infty
$$

с некоторым числом $b>2 \beta$ ( $\beta$ - из леммы 1). Тогда найдется решение задачи (24), (25), удовлетворяющее условию вида (30).

ДокАЗАтЕЛьство. Построим сначала соленоидальный вектор $\boldsymbol{v}(x)$, удовлетворяюший тем же условиям, что и $\boldsymbol{f}(x)$.

Положим $r_{1}=a$; остальные $r_{k}, r=2,3, \ldots$, находятся из условий $r_{k+1}-$ $l\left(r_{k+1}\right)=r_{k}$. Аналогично строится последовательность $\rho_{k}: \rho_{1}=a, \rho_{k+1}-$ $l_{-}\left(\rho_{k+1}\right)=\rho_{k}, k=2,3, \ldots$. Функцию $\boldsymbol{v}^{0} \in \stackrel{\circ}{W}_{2}^{1}\left(\Omega_{-a}^{a}\right)$ выберем из решений уравнения

$$
\operatorname{div} \boldsymbol{v}^{0}=\operatorname{div} \boldsymbol{f}, \quad x \in \Omega_{-a}^{a}
$$

Условие разрешимости этого уравнения выполнено ввиду постоянства потока вектора $\boldsymbol{f}$ через сечения $S_{r}$ :

$$
\int_{\Omega_{-a}^{a}} \operatorname{div} \boldsymbol{f} d x=\int_{S_{a}} \boldsymbol{f} \cdot \boldsymbol{n} d x-\int_{S_{-a}} \boldsymbol{f} \cdot \boldsymbol{n} d x=1-1=0 .
$$

Векторы $\boldsymbol{v}^{k} \in \stackrel{\circ}{\boldsymbol{W}}_{2}^{1}\left(\omega\left(r_{k+1}\right)\right), k=1,2, \ldots$, являются решениями уравнений

$$
\operatorname{div} \boldsymbol{v}^{k}=\operatorname{div} \boldsymbol{f}, \quad x \in \omega\left(r_{k+1}\right)
$$

Векторы $\boldsymbol{v}^{-k}, k=-1,-2, \ldots$, удовлетворяют аналогичным уравнениям в областях $\omega_{-k-1}=\Omega_{-\rho_{k+1}}^{-\rho_{k}}$. Каждый раз выбираются решения, удовлетворяющие неравенствам вида (7), так что справедливы оценки:

$$
\left\|\nabla \boldsymbol{v}^{i}\right\|_{\omega_{i}} \leqslant d\|\operatorname{div} \boldsymbol{f}\|_{\omega_{i}}, \quad i=0, \pm 1, \pm 2, \ldots
$$

где использованы обозначения $\omega_{0}=\Omega_{-a}^{a}, \omega_{k}=\omega\left(r_{k+1}\right), k=1,2, \ldots$ Очевидно, что вектор $\boldsymbol{v}=\boldsymbol{f}-\sum_{i=-\infty}^{\infty} \boldsymbol{v}^{i}$, где функции $\boldsymbol{v}^{i}$ считаются продолженными нулем за пределы своих областей определения, соленоидален, принадлежит пространству $\stackrel{\circ}{\boldsymbol{W}}_{2, \text { lос }}^{1}(\bar{\Omega})$ и удовлетворяет условию вида $(30)$.

Решение задачи (24), (25) будем искать в виде $\boldsymbol{w}=\boldsymbol{u}+\boldsymbol{v}$, где вектор $\boldsymbol{u} \in$ $\stackrel{\circ}{W}_{2, \text { loc }}^{1}(\bar{\Omega})$ имеет нулевой поток через сечения $S_{r}$

$$
\int_{S_{a}} \boldsymbol{u} \cdot \boldsymbol{n} d S=0
$$


и удовлетворяет тождеству

$$
\int_{\Omega}(\nabla \boldsymbol{u}+\nabla \boldsymbol{v}): \nabla \boldsymbol{\eta} d x=0
$$

при любом соленоидальном векторе $\boldsymbol{\eta} \in \boldsymbol{C}_{0}^{\infty}(\Omega)$.

Обозначим через $\stackrel{\circ}{\boldsymbol{H}}(\Omega)$ пополнение множества гладких соленоидальных финитных в $\Omega$ функций по норме $\|\boldsymbol{u}\|_{\boldsymbol{H}}^{2}=\int_{\Omega}|\nabla \boldsymbol{u}|^{2} d x$.

Чтобы построить решение уравнения (31), рассмотрим матричные функции $\boldsymbol{f}^{i}=$ $\chi\left(\omega_{i} ; r_{i-1}, r_{i}\right) \nabla \boldsymbol{v}$, где числа $r_{i}, i=0, \pm 1, \pm 2, \ldots$, определяются следующим образом: $-r_{0}=r_{1}=a$. Остальные вычисляются из индукционного соотношения (27) при положительных $i$ и равенства $r_{i-1}=-l_{-}\left(-r_{i}\right)+r_{i}$ при отрицательных $i$. Векторы $\boldsymbol{u}^{i} \in \stackrel{\circ}{\boldsymbol{H}}(\Omega)$ найдем из уравнений

$$
\int_{\Omega}\left(\nabla \boldsymbol{u}^{i}+\boldsymbol{f}^{i}\right): \nabla \boldsymbol{\eta} d x=0
$$

Они подчиняются оценкам

$$
\left\|\nabla \boldsymbol{u}^{i}\right\| \leqslant\left\|\boldsymbol{f}^{i}\right\|
$$

Введем обозначение $H(r)=\int_{\Omega} \xi\left|\nabla \boldsymbol{u}^{k}\right|^{2} d x$ при некотором фиксированном $k>0$; здесь $\xi(x, r)$ - функция из пункта 2 . Очевидно, $H(r) \leqslant\left\|f^{k}\right\|^{2}$ при всех $r>0$.

ЛЕМма 4. Существует зависящая только от чисел $d$ и $\theta$ постоянная $\mu \in$ $[0, \beta]$ такая, что при всех $r \in\left[a, r_{k}\right]$ справедливо неравенство

$$
H(r) \leqslant C(l(a)) \exp \left(-\int_{r}^{r_{k}} \frac{d \rho}{2 \mu l(\rho)}\right)\left\|f^{k}\right\|^{2}
$$

Доказательство проводится по той же схеме, что и в основной лемме, и поэтому опускается. Приведем лиш промежуточное неравенство, аналогичное (15):

$$
H(r) \leqslant \mu H_{r}(r) l(r)
$$

Далее, как и в доказательстве теоремы 3, при помощи неравенства (32) и условия (30), записанного для функции $\boldsymbol{v}$, доказьвается, что сумма $\sum_{i=-\infty}^{\infty} \boldsymbol{u}^{i}$ сходится в пространстве $\stackrel{\circ}{\boldsymbol{W}}{ }_{2, \text { lос }}(\bar{\Omega})$ к некоторой функции, удовлетворяющей неравенству вида (30). Теорема 4 доказана.

ЗАмечАниЕ. Теорема 4 утверждает сушествование решения задачи $(24),(25)$ с конечным интегралом

$$
\int_{\Omega_{a}}|\nabla \boldsymbol{w}|^{2} \exp \left(-\int_{a}^{x_{1}} \frac{d \rho}{b l(\rho)}\right) d x
$$


которьй имеет вид, отличньй от (26). Однако, для широкого класса областей выполнено соотношение

$$
\sup _{r \geqslant a} \nu^{-1}(r) \exp \left(-\int_{a}^{x_{1}} \frac{d \rho}{2 b l(\rho)}\right)<\infty
$$

в котором $\nu(r)$ обозначает модуль первого собственного значения оператора Лапласа в сечении $S_{r}$ с условием Дирихле на границе. Например, для областей $\Omega$, у которых

$$
\Omega_{a}=\left\{\left|x^{\prime}\right|<x_{1}^{\alpha}, x_{1}>a>0\right\}, \quad \alpha \in(0,1)
$$

очевидно, что $\nu(r)=c(\alpha) r^{-2 \alpha}$, и мы выбираем $l(r)=r^{\alpha}$, так что (33) тривиально.

Пользуясь (33), устанавливаем конечность интеграла

$$
\int_{\Omega_{a}} \nu^{-1}\left(x_{1}\right)|\nabla \boldsymbol{w}|^{2} \exp \left(-\int_{a}^{x_{1}} \frac{d \rho}{2 b l(\rho)}\right) d x
$$

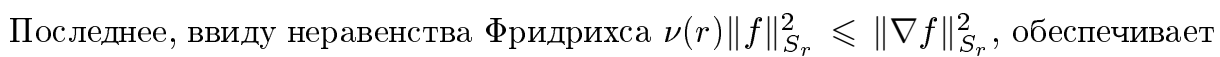
справедливость (26).

6. Для полноты изложения докажем здесь утверждение о div-ограниченности приведенных во введении примеров семейств областей.

Отметим прежде всего, что так как отношение $\|\nabla \boldsymbol{u}\| /\|\operatorname{div} \boldsymbol{u}\|$ не меняется при преобразовании $x \rightarrow r x$, то семейство подобных областей div-ограничено.

В работе [1] предложен оператор

$$
\boldsymbol{u}(x)=\boldsymbol{R}_{r} f=r^{-n} \int_{\mathbb{R}^{n}} f(x-y) \int_{1}^{\infty} h((y+t(x-y)) / r) t^{n-1} d t r y
$$

где $h$ - произвольная гладкая неотрищательная функция с носителем в шаре $B_{1}$ такая, что $\int h d x=1$. Там доказано, что если носитель функции $f \in L_{2}(Q)$ лежит в области $Q$, звездной относительно шара $B_{R}$, и $\int_{Q} f d x=0$, то $\boldsymbol{u} \in \stackrel{\circ}{\boldsymbol{W}}_{2}^{1}(Q)$, $\operatorname{supp} \boldsymbol{u} \subset Q, \operatorname{div} \boldsymbol{u}=f$ и $\sup \left\{\|\nabla \boldsymbol{u}\| /\|f\|: f \in L_{2}(Q)\right\}=d(Q, r)<\infty$. Пусть $R=\sup \{|x|: x \in Q\}$. Очевидно, $d(Q, r) \leqslant d\left(B_{R}, r\right) \equiv g(R, r)$. Легко видеть, что если $\tilde{f}(x)=f(r x)$, то

$$
\frac{\left\|\boldsymbol{R}_{r} f\right\|}{\|f\|}=\frac{\left\|\boldsymbol{R}_{1} \tilde{f}\right\|}{\|\tilde{f}\|}
$$

Отсюда следует однородность функции $g(R, r)=g(R / r, 1)$. Так как $g(R, 1)=$ $\sup \left\{\left\|\nabla \boldsymbol{R}_{1} f\right\| /\|f\|, f \in L_{2}\left(B_{R}\right)\right\}$ - неубывающая функция, то тем самым доказана div-ограниченность семейства равномерно звездных областей.

Покажем теперь, как для области $Q$ с липшицевой границей построить div-ограниченное семейство вложенных подобластей, исчерпывающих область $Q$.

Пусть $Q_{0}$ - подобласть области $Q$, имеющая гладкую границу, лежащую в $\varepsilon$-окрестности границы $\partial Q, \varepsilon>0$. Так как $Q$ - область с липшицевой границей, то 
разность $Q \backslash Q_{0}$ при достаточно малом $\varepsilon$ можно покрыть конечным числом "параллелепипедов" $Q_{i}, Q_{i} \cap Q_{0} \neq \varnothing, i=1, \ldots, m$, имеюших в местной системе координат вид

$$
Q_{i}=\left\{x:\left|x_{j}\right|<\delta / 3 L, j=1, \ldots, n-1,-\delta<x_{n}<f_{i}\left(x^{\prime}\right)\right\}, \quad i=1, \ldots, m .
$$

Здесь число $\delta(\varepsilon)>0, f_{i}-$ некоторые липшицевы функции с постоянной Липшица $L$, удовлетворяющие неравенствам $\delta / 3<f_{i}\left(x^{\prime}\right)<\delta, i=1, \ldots, m$. Легко видеть, что тогда области $Q_{i}^{k}=\left\{x \in Q_{i}: x_{n}<f_{i}\left(x^{\prime}\right)-1 / k\right\}$ равномерно звездны при натуральных числах $k>3 / \delta, i=1, \ldots, m$, так что они вместе с $Q_{0}$ образуют div-ограниченное семейство.

Докажем, что области

$$
\Omega_{k}=Q_{0} \cup\left(\bigcup_{i=1}^{m} Q_{i}^{k}\right), \quad k>3 / \delta,
$$

также образуют div-ограниченное семейство. Зафиксируем $k>3 / \delta$. Пусть $\chi_{0}, \chi_{i}$ - характеристические функции множеств $Q_{0}, Q_{i}^{k}, i=1, \ldots, m$. Положим

$$
\varphi(x)=\sum_{i=0}^{m} \chi_{i}(x), \quad \varphi_{i}=\chi_{i} / \varphi, \quad i=0, \ldots, m .
$$

Пусть $\psi_{i}(x)$ - некоторые неотрицательные функции с носителями, лежащими в множествах $Q_{0} \cap Q_{i}^{k}, i=1, \ldots, m$, такие, что $\int \psi_{i} d x=1$.

Возьмем произвольную функцию $f \in L_{2}\left(\Omega_{k}\right), \int_{\Omega_{k}} f d x=0$, и рассмотрим уравнения

$$
\operatorname{div} \boldsymbol{u}^{i}=f \varphi_{i}-c_{i} \psi_{i}, \quad x \in Q_{i}^{k}, \quad i=1, \ldots, m,
$$

в которых $c_{i}$ находятся из условия разрешимости $c_{i}=\int_{Q} f \varphi_{i} d x$. Эти уравнения имеют решения $\boldsymbol{u}^{i} \in \stackrel{\circ}{\boldsymbol{W}}_{2}^{1}\left(Q_{i}^{k}\right)$, подчиняюшиеся оценкам

$$
\left\|\nabla \boldsymbol{u}^{i}\right\| \leqslant d\left\|f \varphi_{i}-c_{i} \psi_{i}\right\| \leqslant d\|f\|\left(1+\left\|\varphi_{i}\right\|\left\|\psi_{i}\right\|\right) .
$$

Ввиду условия

$$
\sum_{i=0}^{m} \int_{Q} f \varphi_{i} d x=0
$$

уравнение $\operatorname{div} \boldsymbol{u}^{0}=f \varphi_{0}+\sum_{i=1}^{m} c_{i} \psi_{i}$ также имеет решение, удовлетворяюшее оценке

$$
\left\|\nabla \boldsymbol{u}^{0}\right\| \leqslant d\left\|f \varphi_{0}+\sum_{i=1}^{m} c_{i} \psi_{i}\right\| \leqslant d\|f\|\left(1+\sum_{i=1}^{m}\left\|\varphi_{i}\right\|\left\|\psi_{i}\right\|\right) .
$$

Очевидно, вектор $\boldsymbol{u}=\sum_{i=0}^{m} \boldsymbol{u}^{i}$ принадлежит $\stackrel{\circ}{\boldsymbol{W}_{2}^{1}}\left(\Omega_{k}\right)$, является решением уравнения $\operatorname{div} \boldsymbol{u}=f$ и подчиняется оценке $\|\nabla \boldsymbol{u}\|_{\Omega_{k}} \leqslant c d\|f\|$ с постоянной $c$, которую можно выбрать не зависяшей от $k$. 


\section{Список литературы}

1. Боговский M.E. Решение некоторых задач векторного анализа, связанных с операторами div и grad // Труды семинара С.Л. Соболева. №1. Новосибирск: Наука, 1980. C. $5-40$.

2. Гущин $A . K$. О равномерной стабилизации решений второй смешанной задачи для параболического уравнения // Матем. сб. 1982. Т. 119. С. 451-508.

3. Heywood J. G. On uniqueness questions in the theory of viscous flow // Acta Math. 1976. V. 138. P. 61-102.

4. Лады женская $O$. А. Математические вопросы динамики вязкой несжимаемой жидкости. М.: Наука, 1970.

5. Ладыженская $O . A$, Солонников $B . A$. О начально-краевой задаче для линеаризованной системы уравнений Навье-Стокса в областях с некомпактными границами // Труды МИАН. 1983. Т. 159. С. 37-40.

6. Мукминов $\Phi . X$. О равномерной стабилизации решений внешней задачи для уравнений Навье-Стокса // Матем. сб. 1994. Т. 185. № 3. С. 41-68.

7. Олейник O.A., Иосифьлн Г.А. Аналог принципа Сен-Венана и единственность решений краевых задач в неограниченных областях для параболических уравнений // УМН. 1976. T. 31. C. $142-166$.

8. Tacklind $S$. Sur les classes quasianalytiques des solutions des equations aux derivees partielles du type parabolique // Nova Acta Reg. Soc. Sci. Upsaliensis. Ser. 4. 1936. V. 10. № 3 .

9. Borchers $W$., Sohr $H$. The equations $\operatorname{div} \boldsymbol{u}=f$ and $\operatorname{rot} \boldsymbol{v}=g$ with homogeneous Dirichlet boundary condition // Hokkaido Math. J. 1990. V. 19. P. 67-87.

Башкирский государственнњй университет, Уфа

Поступила в редакцию

E-mail: root@bgua.bashkiria.su; yavdat@bgua.bashkiria.su 30.01.1995 